\title{
Altération du gène codant pour la perforine dans la lymphohistiocytose familiale
}

$\mathbf{O}^{\prime}$ n connaît depuis plusieurs années le rôle clé que joue la perforine dans l'activité cytotoxique déployée par les cellules tueuses, lymphocytes $\mathrm{T}$ et cellules de l'immunité naturelle (natural killer) [1]. La perforine, et d'autres effecteurs de la cytotoxicité, comme les granzymes A et B, sont contenus dans un même compartiment intracellulaire, les granules cytotoxiques des cellules tueuses $\left(\mathrm{m} / \mathrm{s} 1995, n^{\circ} 11\right.$, p. 99-104). Perforine et granzymes sont les effecteurs d'un même mécanisme de mort qui est utilisé par la cellule cytotoxique, dès qu'elle identifie une cellule menaçante pour l'organisme, qu'elle soit étrangère, infectée ou tumorale. La perforine déversée dans l'espace intermembranaire par la cellule cytotoxique, lors de son contact avec la cellule cible et de sa reconnaissance, perfore la membrane cytoplasmique de la cellule cible en de multiples points. Elle ouvre ainsi la voie aux granzymes qui pénètrent dans la cellule, migrent dans le noyau et entraînent une apoptose au moins en partie dépendante de la voie des caspases. Ce mécanisme de cytotoxicité en trans (dirigé contre les cellules présentatrices d'antigène ou CPA), qui requiert l'exocytose des granules, est, semble-t-il, essentiel dans la réponse antivirale. Au contraire, l'autre voie cytotoxique utilisée par ces mêmes cellules tueuses, et qui implique les molécules Fas et FasL, semble intervenir plus particulièrement dans le maintien de l'homéostasie du système immunitaire. La responsabilité de la perforine dans l'expression d'une maladie héréditaire du système immunitaire, la lymphohistiocytose familiale (LHF), démontre aujourd'hui que, à côté de la voie Fas/FasL, la perforine joue également un rôle crucial chez l'homme dans le rétrocontrôle de la réponse immune qui a su se mobiliser pour combattre une infection.

La lymphohistiocytose familiale (LHF) est une maladie héréditaire extrêmement sévère du système immunitaire, transmise selon un mode autosomique récessif. La seule thérapeutique actuellement efficace est la greffe de moelle osseuse. La LHF se manifeste tôt dans l'enfance, souvent au décours d'une infection virale, et se caractérise classiquement par une prolifération polyclonale de lymphocytes $\mathrm{T}$ activés, essentiellement de phénotype $\mathrm{CD}^{+}$. Ces lymphocytes activés infiltrent les organes lymphoïdes primaires, secondaires puis progressivement tous les organes y compris le système nerveux central. Des macrophages activés participent également à cet infiltrat. Des images d'hémophagocytose (sous forme de macrophages activés ayant phagocyté un ou plusieurs lymphocytes ou d'autres cellules hématopoiétiques) sont observées, en particulier dans la moelle osseuse. L'activation lymphocytaire et macrophagique se traduit également par la sécrétion, par ces cellules, d'importantes quantités de cytokines, en particulier d'INF $\gamma$ (interféron $\gamma$ ) et de TNF $\alpha$ (tumor necrosis factor $\alpha$ ), dont les actions rendent comptent des divers éléments du tableau clinique et biologique observé. L'effet, au moins transitoire, des traitements lymphotoxiques suggère que les lymphocytes $\mathrm{T}$ jouent un rôle décisif dans la genèse de ce syndrome d'activation lymphocytaire et macrophagique.
$\mathrm{Au}$ cours de ces dernières années, plusieurs équipes ont tenté de localiser le gène responsable de cette affection. Les difficultés auxquelles elles se sont heurtées ont fait envisager une hétérogénéité génétique de cette maladie, que le phénotype ne suggérait pas. Au cours de l'année 1999, deux équipes ont identifié deux régions chromosomiques différentes toutes deux liées à la LHF. Une équipe britannique identifiait un locus sur le chromosome 9 en 9q21-q22.3 [2], locus dont l'association semble restreinte à un groupe de familles pakistanaises. Notre équipe apportait la démonstration statistique de l'hétérogénéité de la maladie et identifiait un locus sur le chromosome 10 en 10q21-q22 [3]. Mais l'hétérogénéité de la maladie s'avère plus grande encore puisque dans près de la moitié des familles, la ségrégation de la lymphohistiocytose familiale n'est compatible avec aucune de ces deux localisations. L'intervalle génétique identifié sur le chromosome 10 contenait, parmi d'autres gènes, celui codant pour la perforine. Les souris dont le gène de la perforine a été invalidé (per-/-), présentent un défaut isolé d'activité cytotoxique, et vivent parfaitement en élevage non protégé. Cependant, ces souris ont une susceptibilité particulière à certaines souches du virus LCMV (virus de la chorioméningite lymphocytaire ) [4], et meurent dans un contexte de lymphoprolifération [5] rappelant le phénotype LHF. La perforine devenait alors un candidat fort, cumulant le statut de candidat fonctionnel à celui de candidat positionnel. L'étude du gène chez plusieurs enfants atteints de LHF nous a permis d'attribuer à la perforine la 
responsabilité de cette maladie [6]. Des mutations non sens homozygotes ont été identifiées chez quatre patients non apparentés et des mutations faux sens chez quatre autres. Dans tous les cas, ces mutations entraînent une expression extrêmement diminuée ou même indétectable de la perforine dans les granules cytotoxiques, et, en conséquence, un défaut de l'activité cytotoxique induite lors de l'exocytose de ces granules. En revanche, il n'y a aucune altération de la cytotoxicité contrôlée par la voie Fas/FasL. Le gène codant pour la perforine est donc le premier gène mis en cause dans la LHF.

Quelles informations la LHF et le modèle murin perf ${ }^{-}$nous fournissent-ils quant au rôle de la perforine dans la régulation de l'activation lymphocytaire? Les différentes données dont nous disposons à ce jour suggèrent le modèle suivant (figure 1): lors d'une infection virale, un défaut de perforine, et l'altération de l'activité cytotoxique des lymphocytes qui en résulte, expliquerait la persistance des cellules présentatrices de l'antigène (CPA) dans l'organisme, et donc la perpétuation de l'activation de la réponse immune [5]. En réponse à cette stimulation permanente, les lymphocytes T cytotoxiques mobilisés sécrètent de grandes quantités d'IFN $\gamma$ qui induisent la synthèse de cytokines proinflammatoires (TNF $\alpha$, IL-1, IL-6...) par les macrophages. Ainsi s'amorcent des boucles paracrines et autocrines dont les effets délétères expliquent les divers éléments du tableau clinique et biologique. Ce modèle, déduit d'une action en trans de la perforine (action sur les cellules CPA qui ne sont pas les cellules productrices), est compatible avec l'observation d'un contrôle de la maladie après greffe de moelle osseuse même si le chimérisme est peu important [7]. Dans cette situation, en effet, les cellules cytotoxiques saines issues du donneur, peuvent, même si elles sont en nombre réduit, tuer successivement plusieurs CPA. Parallèlement, la perforine semble être capable d'une action en cis [8], permettant l'élimination des cellules activées.

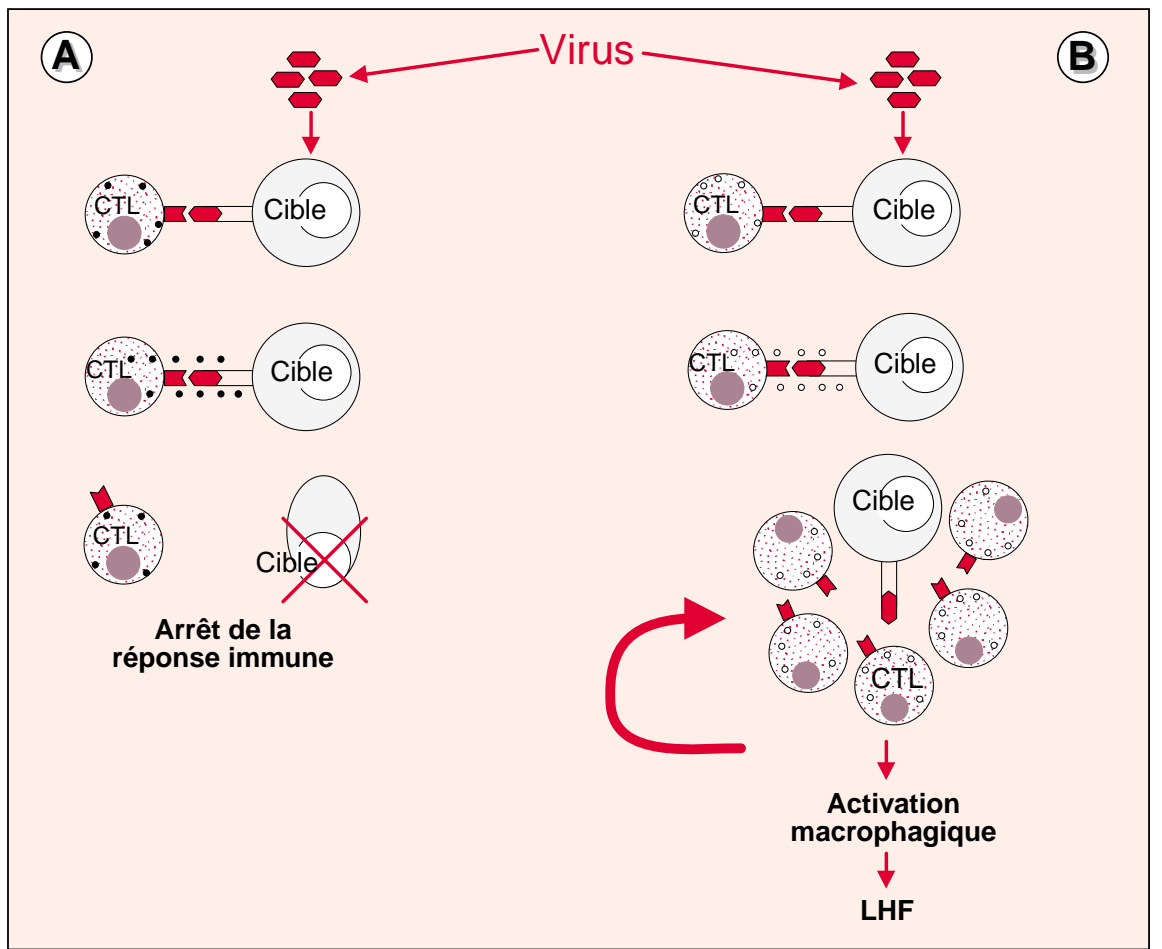

Figure 1. Schéma illustrant l'action de la perforine lors d'une réponse immune antivirale et les conséquences de son altération. En situation normale (A) les granules cytotoxiques contiennent de la perforine (cercles noirs) et le déversement de leur contenu élimine la cible CPA, ce qui aboutit à l'arrêt de la réponse immune. En l'absence de perforine (B) dans les granules cytotoxiques (schématisés par des cercles blancs), la cible (CPA) n'est pas détruite, ce qui pérennise l'activation et l'amplification des lymphocytes $T$ activés, induit une activation macrophagique, qui aggrave l'activation lymphocytaire, et conduit au tableau de la LHF.

lymphocytes $\mathrm{T}$ activés et leur action délétère. Ce mécanisme participerait ainsi au phénotype de la maladie.

Le phénotype sévère de la LHF diffère de celui qui est observé chez les patients présentant un défaut d'apoptose lié à la molécule Fas [9] $\left(\mathrm{m} / \mathrm{s} 1995, n^{\circ} 11, p .1180\right)$, ou de celui des modèles murins équivalents ( $l p r$ et $g l d)\left(m / s ~ 1992, n^{\circ} 7, p .735\right)$. Dans ces situations, on note une lymphoprolifération importante de cellules $\mathrm{T}$ doubles négatives $\left(\mathrm{CD} 4^{-} \mathrm{CD} 8^{-}\right)$ dans le cadre d'une affection chronique, à laquelle s'associent des manifestations auto-immunes.

La LHF n'est pas le seul modèle humain caractérisé par l'apparition d'un syndrome d'activation lymphocytaire et macrophagique. Un syndrome identique est observé au cours de trois autres maladies héréditaires, le syndrome de Chediak-Higashi $(\mathrm{m} / \mathrm{s}$
1999, $\left.n^{\circ} 15, p .1292\right)$, le syndrome de Griscelli et le syndrome de Purtilo [10]. De façon tout à fait intéressante, les gènes responsables des deux premiers syndromes codent pour des protéines participant au trafic intracellulaire, et leur anomalie entraîne un défaut de la libération du contenu des granules cytotoxiques. Ces granules contiennent la perforine et également d'autres molécules immunorégulatrices telles que CTLA-4 [11] $\left(\mathrm{m} / \mathrm{s}\right.$ 1996, $n^{\circ} 8-9$, p. 990) et FasL conférant au granule cytotoxique un rôle déterminant dans la régulation de la réponse immune. L'identification de la perforine comme un des gènes responsables de la LHF permet de faire l'hypothèse que d'autres molécules, nécessaires à cette activité cytotoxique ou au contraire l'inhibant, soient impliquées dans les autres 
formes héréditaires ou acquises de ces syndromes d'activation lymphocytaires et macrophagiques, ouvrant par là même de nouvelles perspectives pour l'exploration de ces maladies

\section{RÉFÉRENCES}

1. Vély F, Vivier E. Mécanismes moléculaires de la cytotoxicité des cellules NK. Med Sci $1996 ; 12$ : 458-64.

2. Ohadi M, Lalloz MR, Sham P, et al. Localization of a gene for familial hemophagocytic lymphohistiocytosis at chromosome 9q21.3-22 by homozygosity mapping. Am J Hum Genet 1999; 64: 165-71.

3. Dufourcq-Lagelouse R, Jabado N, Le Deist $\mathrm{F}$, et al. Linkage of familial hemophagocytic lymphohistiocytosis to 10q21-22 and evidence for heterogeneity. Am J Hum Genet $1999 ; 64: 172-9$.

4. Kägi D, Ledermann B, Bürki K, et al. Cytotoxicity mediated by $\mathrm{T}$ cells and natural killer cells is greatly impaired in perforindeficient mice. Nature 1994; 369: 31-7.
5. Matloubian M, Suresh M, Glass A, et al. A role for perforin in downregulating $\mathrm{T}$-cell response during chronic viral infection. $J$ Virol 1999; 73: 2527-36.

6. Stepp SE, Dufourcq-Lagelouse R, Le Deist F, et al. Perforin gene defects in familial hemophagocytic lymphohistiocytosis. Science 1999 ; 286 : 1957-9.

7. Landman-Parker J, Le Deist F, Blaise A, Brison $\mathrm{O}$, Fischer A. Partial engraftment of donor bone marrow cells associated with long-term remission of haemophagocytic lymphohistiocytosis. Br J Haematol 1993; 85 : $37-41$.

8. Spaner D, Raju K, Rabinovitch B, Miller RG. A role for perforin in activation-induced cell death in vivo: increased expansion of allogenic perforin deficient $\mathrm{T}$ cell in SCID mice. J Immunol 1999; 162: 1192-9.

9. Rieux-Laucat F, Le Deist F, Hivroz C, et al. Mutations in Fas associated with human lymphoproliferative syndrome and autoimmunity. Science 1995; 268: 1347-9.

10. Dufourcq-Lagelouse R, Pastural E, Barrat FJ, et al. Genetic basis of hemophagocytic lymphohistiocytosis syndrome. Int $J \mathrm{Mol}$ Med 1999; 4 : 127-33.
11. Barrat FJ, Le Deist F, Benkerrou M, et al. Defective CTLA-4 cycling pathway in Chediak-Higashi syndrome: a possible mechanism for deregulation of T lymphocyte activation. Proc Natl Acad Sci USA 1999; 96 : $8645-50$

\section{TIRÉS À PART}

G. de Saint Basile.

\section{Rémi Dufourcq Lagelouse \\ Françoise Le Deist \\ Alain Fischer \\ Geneviève de Saint Basile}

Inserm U. 429, Développement normal et pathologique du système immunitaire, Pavillon Kirmisson, Hôpital NeckerEnfants-malades, 149, rue de Sèvres, 75015 Paris, France. 